\title{
La nanochirurgie laser en biologie cellulaire
}

$>$ La cellule est un univers dynamique et compartimenté où interagissent une multitude de sous composants à l'échelle nanométrique. Afin d'étudier l'organisation subcellulaire, il est devenu nécessaire de posséder des outils permettant une manipulation directe extrêmement précise et non invasive. L'avènement des lasers à impulsions, dès les années 60 , a conduit à la naissance de la chirurgie au laser. Aujourd'hui, la réduction des impulsions laser en dessous de la nanoseconde permet de mieux comprendre leur interaction avec les tissus biologiques et de contrôler des interventions chirurgicales à une résolution de l'ordre de quelques centaines de nanomètres. Utilisant l'ionisation de la matière par la lumière, cette nanochirurgie laser permet d'effectuer des interventions chirurgicales intracellulaires telles que la découpe de microtubules ou de fibres de tension, sans endommager les structures environnantes ou compromettre la viabilité cellulaire. Ainsi, l'utilisation de lasers à impulsions ultra-courtes, plus précis et puissants, offre une nouvelle approche pour l'étude des forces en biologie ou pour la quantification de la dynamique du cytosquelette. $<$
Julien Colombelli, Rainer Pepperkok, Ernst H.K. Stelzer, Emmanuel G. Reynaud

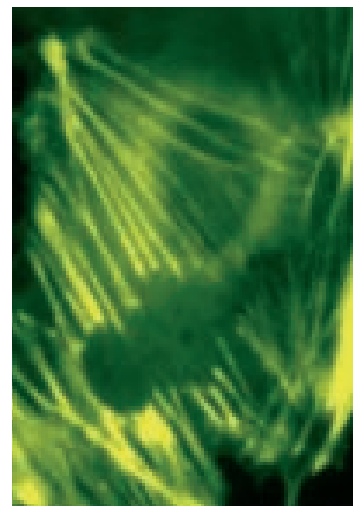

Laboratoire Européen de Biologie Moléculaire (LEBM), Meyerhofstrasse 1, D-69117 Heidelberg, Allemagne. colombel@embl.de

l'environnement cellulaire direct pour une utilisation sur des structures biologiques vivantes.

La chirurgie au laser de tissus biologiques a été étudiée et appliquée depuis plus de quarante ans, essentiellement depuis l'invention des lasers pulsés. De nombreux lasers aux capacités techniques très variables ont été employés dans ce but et il est difficile pour le non spécialiste de distinguer leurs limites. En comprimant la durée des impulsions lumineuses à des temps extrêmement brefs de l'ordre de la nanoseconde (l $\mathrm{ns}=10^{-9} \mathrm{~s}$ ) jusqu'à atteindre quelques dizaines de femtosecondes ( $1 \mathrm{fs}=10^{-15} \mathrm{~s}$ ) pour les lasers les plus modernes, la puissance maximale de chaque impulsion peut être augmentée de plusieurs ordres de grandeur tout en conservant une énergie raisonnablement basse. On peut ainsi induire des effets non linéaires dans le milieu irradié allant de la dégradation photochimique à la pure explosion mécanique et thermale [1-3]. En focalisant en un point précis a l'intérieur d'un tissu ou sous la membrane d'une cellule, il est possible de vaporiser localement le matériel intracellulaire sans que l'effet photodestructeur ne s'étende au delà de quelques centaines de nanomètres autour de la cible irradiée. On peut ainsi découper précisément le cytosquelette $[4,5]$ (microtubules, microfilaments d'actine) ou des organelles telle qu'une mitochondrie [6] sans altérer les structures environnantes ou compromettre la viabilité cellulaire. Cette technique non invasive permet une manipulation directe de la machinerie cellulaire à une résolution inférieure au micromètre d'où le terme de nanochirurgie (nanosurgery).

Article reçu le 5 janvier 2006, accepté le 15 mars 2006. 
Ce dossier couvrira en premier lieu les principes physiques de la dissection laser ainsi que leurs limitations techniques et physiques afin de montrer comment les récents développements technologiques ont permis la réduction de l'extension spatiale et temporelle de l'effet chirurgical. La deuxième partie présentera les différentes applications récentes de la nanochirurgie en biologie cellulaire et du développement, en particulier pour l'étude des forces. II n'existe, à l'heure actuelle, aucune définition internationale permettant de distinguer les différents niveaux de précision en chirurgie laser. Donc, pour faciliter la lecture, nous emploierons le terme microchirurgie pour les utilisations chirurgicales classiques en ophtalmologie, cardiologie, urologie et biologie du développement, le terme microdissection pour l'application particulière des lasers à l'isolation de tissus spécifiques en pathologie et le terme de nanochirurgie pour les applications submicrométriques en biologie cellulaire.

\section{Principes physiques de la nanochirurgie laser}

L'interaction laser-tissu se décompose en plusieurs phénomènes complexes et souvent intriqués: les actions photothermique, photoablative et électromécanique. Chacun de ces phénomènes est la résultante d'une dose d'énergie optique absorbée par le tissu pendant la durée de l'émission et de nombreux effets collatéraux se propagent au-delà de cette période.

L'action photothermique est obtenue avec des impulsions de $1 \mathrm{~ms}$ à quelques secondes et des irradiances de 10 à $10^{6}$ Watt $/ \mathrm{cm}^{2}$. II en résulte une élévation de chaleur tissulaire. Son amplitude et sa durée sont responsables de la dénaturation cellulaire et des protéines et les applications médicales et thérapeutiques sont nombreuses $[2,3]$. L'action photochimique est obtenue avec des temps d'exposition de la dizaine de secondes à la dizaine de minutes avec des irradiances très faibles. On excite ainsi un photosensibilisant appliqué ou injecté qui, une fois activé, va produire des effets létaux. L'action photoablative peut s'obtenir avec des impulsions de 10 à 100 nanosecondes. Elle est la conséquence de réactions photochimiques impliquant l'absorption de photons à haute énergie [7], typiquement dans le domaine de l'ultraviolet (UV), suffisamment élevée pour atteindre l'énergie de dissociation des molécules et, de ce fait, briser les liaison chimiques. Cela entraîne la photodécomposition des molécules irradiées. Cependant, les effets secondaires comme les mutations génétiques ou l'aspect peu pratique de la lumière UV en microscopie conventionnelle sont des facteurs limitant la flexibilité d'application de cet effet. En revanche, l'action électromécanique qui est obtenue avec des impulsions très courtes (100 fs à $10 \mathrm{~ns}$ ) et des irradiances extrêmement élevées ( $10^{7}$ à $10^{12}$ $\mathrm{W} / \mathrm{cm}^{2}$ ) menant a la rupture mécanique du milieu par «claquage optique » ou induction d'un plasma, est plus versatile.

\section{Ionisation de la matière par la lumière}

La formation d'un plasma [1] de dimensions nanométriques survient lors de la focalisation d'impulsions très courtes au travers d'objectifs à grande ouverture numérique $(>0,9)$ qui provoquent l'ionisation de la matière ( $\varepsilon$ ncadré). Ce phénomène, qui débute par la libération d'un électron par absorption de plusieurs photons, se reproduit en cascade pendant toute la durée de l'impulsion laser, provoquant une avalanche d'électrons libres et décomposant ainsi la matière, formant le plasma. La formation d'un plasma survient au-delà d'une énergie de seuil. De manière qualitative, le plasma s'étend d'autant plus que l'énergie fournie dépass e l'énergie de seuil pendant toute la durée de l'impulsion. Aussitôt formé, le plasma absorbe une partie de la lumière et peut provoquer certains effets secondaires tels que la propagation d'une onde de choc ou la vaporisation du milieu par cavitation, autant de phénomènes qui contribuent à amplifier l'extension spatiale et temporelle de l'effet chirurgical. Ainsi, les récents développements technologiques des lasers ont permis la réduction de son extension spatiale et temporelle. En focalisant étroitement des impulsions ultracourtes, la puissance

\section{LA FORMATION DU PLASMA :}

\section{IONISATION DE LA MATIEेRE PAR LA LUMIÈRE}

La réaction induisant l'ionisation d'un milieu exposé à une impulsion laser se divise en deux phases (Figure A). En premier lieu, l'absorption simultanée de plusieurs photons incidents provoque la libération d'un électron, ou électron quasi libre. Cet effet prend le nom d'ionisation à multiphoton et souvent ne suffit pas à obtenir l'effet chirurgical désiré du fait de la trop courte portée des électrons libérés. Cependant, ces électrons peuvent absorber d'autres quantités d'énergie, ou photons, provenant de la même impulsion laser en interagissant avec les atomes voisins. Ce faisant, un électron libre acquiert une énergie cinétique, ou vitesse, suffisante pour provoquer la libération d'un autre électron par impact avec une molécule. L'ionisation d'impact qui en résulte produit donc deux électrons libres qui pourront être de nouveau accélérés par absorption de nouveaux photons. Le phénomène d'impact peut ainsi se reproduire en cascade pendant toute la durée de l'impulsion laser, provoquant une avalanche d'électrons libres qui forment le plasma. La formation d'un plasma survient au delà d'une énergie de seuil (ou puissance de seuil intégrée dans le temps) (Figure B). Des données expérimentales recueillies dans une revue récente [1] (Figure C) montrent, que dans un milieu aqueux, cette intensité de seuil augmente de 3 ordres de grandeur lorsque la durée d'impulsion diminue de $10 \mathrm{~ns}$ à $100 \mathrm{fs}$. Cependant, l'énergie déposée n'est autre que la puissance multipliée par la durée d'impulsion et, par conséquent, il faut 100 fois moins d'énergie pour produire un plasma en utilisant des impulsions ultra-courtes. C'est donc autant d'énergie qui ne sera pas simultanément absorbée par le plasma. Les Figures $B$ et $D$ représentent l'évolution temporelle et spatiale d'un plasma en trois temps, de sa formation à son extension maximale. De manière qualitative, le plasma s'étendra d'autant plus que l'énergie fournie dépasse l'énergie de seuil. Pour visualiser l'extension spatiale d'un plasma, il suffit de focaliser une impulsion laser dans du verre. La formation d'un plasma change les propriétés optiques du verre et il devient visible en transmission. La Figure $\varepsilon$ montre l'extension d'un plasma induit par une impulsion de 470 ps [8] à l'intérieur du verre pour l'énergie de seuil et au-delà de cette énergie où se crée une onde de choc qui fracture la structure du verre bien au-delà du volume d'action de l'impulsion. Ce faisant, nous avons démontré l'extension spatiale d'un plasma mais aussi le caractère tridimensionnel du procédé qui survient exactement au foyer optique et s'étend à partir de ce point, comme illustré dans la Figure F où une pyramide formée de carrés concentriques dans des plans différents a été gravée à l'intérieur du verre. D'autres auteurs ont obtenu des précisions bien en dessous de la limite de diffraction, jusqu'à 30 nm [9], en utilisant des impulsions ultra-courtes de 800 fs. 

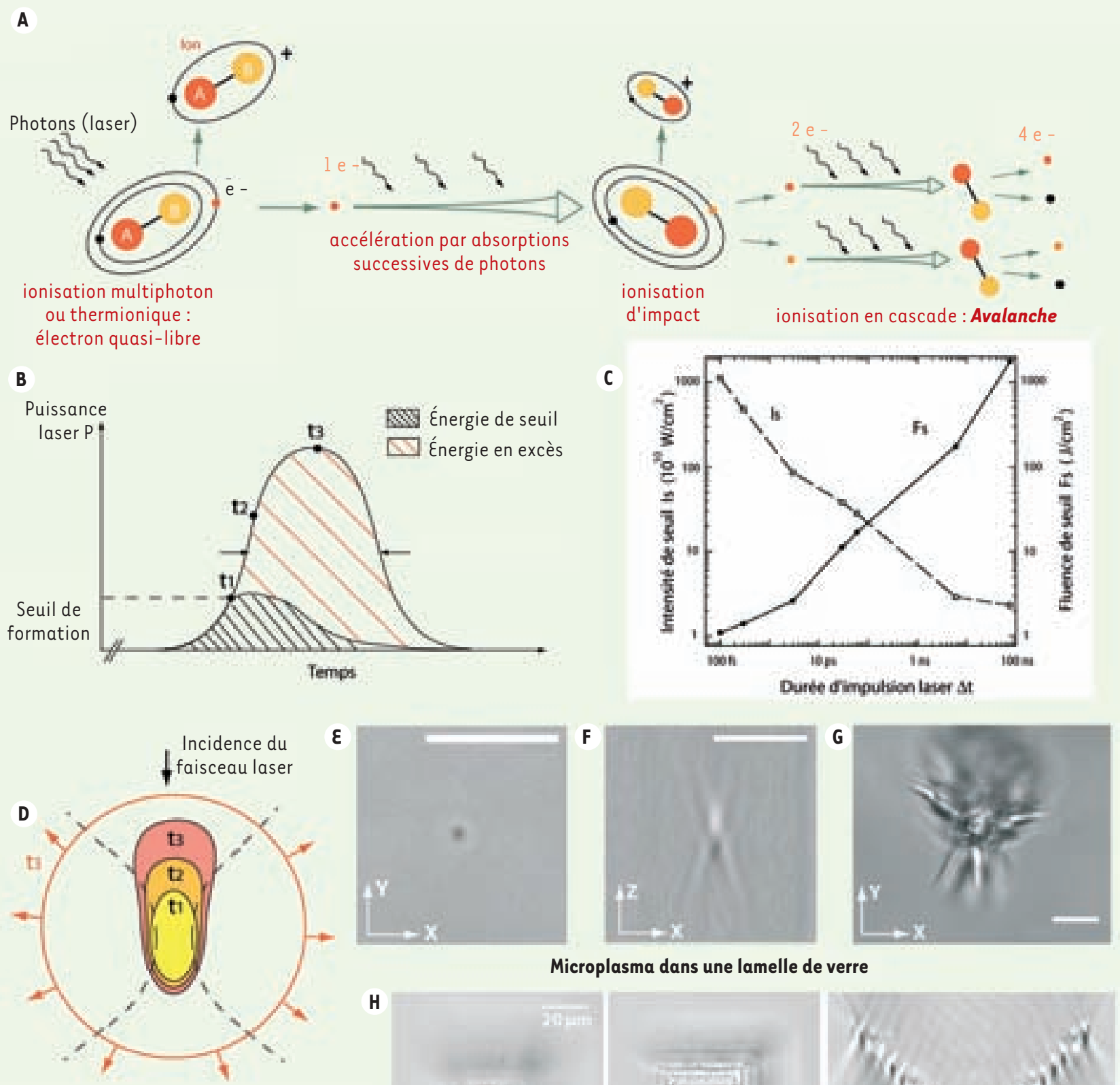

Microplasma dans une lamelle de verre
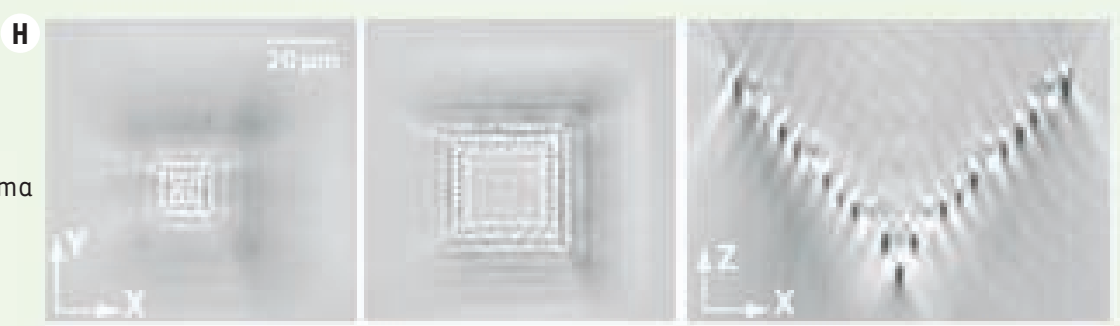

tl : Ionisation du milieu, formation du plasma

t2: Expansion spatiale du plasma vers la source laser

t3 : Expansion maximale accompagnée d'effets secondaires

(onde de choc, cavitation)

Localisation en 3D

Figure de l'Encadré. Dynamique de formation d'un plasma. La matière est représentée par de simples molécules (A-B) pour simplifier la représentation. A. Séquence de libération et d'accélération d'électrons libres. Un nuage d'électrons libres, ou plasma, se forme par ionisation en cascade au-delà d'un certain seuil d'énergie. B. Si la durée d'impulsion diminue, le seuil d'énergie diminue. C. Lorsque l'énergie fournie au milieu dépasse le seuil, le plasma peut s'étendre le long de la direction d'incidence du faisceau laser. $D$. Au temps tl, le plasma se forme et occupe un volume initial (en jaune), puis s'étend en $\mathrm{t} 2$ jusqu'à atteindre une extension maximale en $\mathrm{t} 3$. Engendré dans du verre, le plasma change les propriétés optiques du milieu. $\varepsilon$ n $\varepsilon-G$, la forme du plasma au seuil d'énergie (par une impulsion UV de 470 ps, $\varepsilon-F$ ) et la fracture du verre pour une énergie en excès (G). H. Pyramide en 3D formée de carrés concentriques dans des plans différents, vue en transmission et projetée axialement. $B, C$ et $D:$ adaptées avec permission (d'après [10]); $\varepsilon$ et $F$, (d'après [8]). Échelle $\varepsilon: 5 \mu \mathrm{m}$. 
nécessaire à la formation d'un plasma est atteinte avec beaucoup moins d'énergie qu'avec des lasers utilisés précédemment. Ce faisant, la durée de vie et les dimensions du plasma sont réduites [1].

\section{Quel laser pour quel effet?}

Définir la limite entre un effet chirurgical induit par un plasma pur ou par les effets secondaires qui lui succèdent est un point de discussion depuis plusieurs années. Vogel et al. ont apporté récemment une réponse au problème en simulant les effets de lasers à femtosecondes dans un milieu aqueux. En utilisant une fréquence de répétition différente (le nombre d'impulsions par seconde), ils démontrent que l'effet ablatif d'un laser à femtosecondes peut être induit par
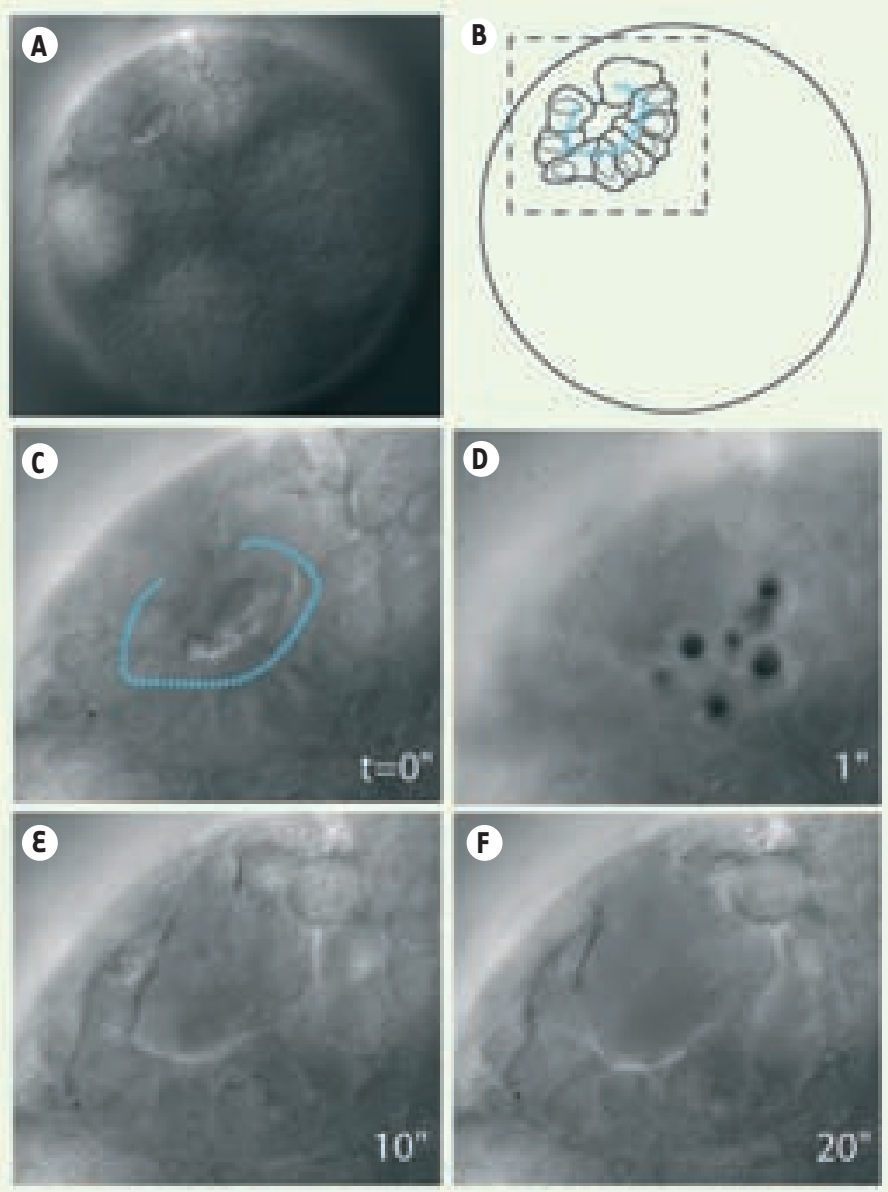

Figure 1. Ablation cellulaire dans un embryon de $P_{*}$ dumerilii. Une structure cellulaire précurseur du développement de la bouche de l'animal adulte, ou Stomodeum, se forme au stade embryonnaire à 25 h (rosette en $\boldsymbol{A}$, schématisée en $\boldsymbol{B}$ ). Le foyer du faisceau laser est balayé le long de ces cellules (pointillés bleus) à l'aide de miroirs pivotants identiques à ceux utilisés en microscopie confocale. C. Vue agrandie des cellules ciblées correspondant à l'aire en pointillé en $B$. La perméabilisation cellulaire est induite par plasma et cavitation (bulles en $\boldsymbol{D}$ ), conduisant à la disparition des cellules visées en quelques secondes, après 10 et 20 secondes en $\varepsilon$ et $\boldsymbol{F}$. Ici la cavitation est induite avec une énergie égale à deux fois l'énergie nécessaire à former un plasma (durée d'impulsion 470 ps). Diamètre de l'embryon : 0,15 mm. pur effet plasma à très basse énergie avec des trains d'impulsions à 80 mégaHertz (80 millions par seconde) [10]. En revanche, avec le même laser, mais cette fois calibré pour donner des trains d'impulsions au kiloHertz, un effet ablatif comparable est atteint à plus haute énergie par création d'une bulle de cavitation atteignant un diamètre d'environ $300 \mathrm{~nm}$ qui, une fois formée, s'écroule sous la pression du milieu environnant en créant une onde de choc très localisée. L'effet cumulatif d'un train d'impulsions très rapide contribue donc à améliorer l'efficacité de la nanochirurgie en limitant le stress thermo-élastique et les études les plus récentes appliquées à la chirurgie intracellulaire rapportent effectivement des interventions utilisant plusieurs centaines à plusieurs millions d'impulsions lasers à très hautes fréquences pour découper le cytosquelette [5, 11, 12], une mitochondrie [6] ou des chromosomes [13].

D’une manière générale, il n'existe pas de laser parfait en nanochirurgie. Cependant, plusieurs paramètres optiques ne peuvent être négligés. La qualité du faisceau doit être irréprochable. Un laser ultraviolet à gaz, utilisé, par exemple, dans certains systèmes de microdissection pour tissus pathologiques, ne peut être focalisé au minimum dû à la pauvreté du profil du faisceau, ce qui provoque en général d'importants effets secondaires thermiques et mécaniques. L'extension du foyer détermine l'intensité du faisceau et, par conséquent, l'ouverture numérique de l'objectif doit être maximale, au moins supérieure à 0,9 . En terme de longueur d'onde, l'utilisation de rayons ultraviolets UV-A (proches du spectre visible) est le meilleur compromis pour réduire l'extension du foyer, proportionnel à la longueur d'onde. II est important de considérer que l'effet initiateur d'un plasma, l'ionisation à multiphoton, est possible de l'ultraviolet à l'infrarouge. Cependant, la pénétration optique des UV dans les tissus biologiques ne dépasse pas la centaine de microns et c'est pourquoi l'utilisation de laser dans l'infrarouge ou le visible est plus efficace dans des tissus profonds, comme par exemple en biologie du développement.

La nanochirurgie peut être donc optimisée en utilisant des trains d'impulsions ultra-courtes en dessous de la nanoseconde. Cela explique le récent succès des lasers à femtosecondes en chirurgie au laser car l'utilisation d'impulsions ultra courtes, en dessous de la picoseconde, permet un meilleur contrôle de l'effet désiré et de son extension spatiale. Néanmoins, l'utilisation combinée du même laser à des fins chirurgicales et d'imagerie, une tendance récente, comporte le risque de compromettre l'intégrité du spécimen, étant donné que les seuils de nanochirurgie plasma et les niveaux d'énergie utilisés pour observer en microscopie non linéaire (à multiphoton) sont très proches [10].

\section{Applications}

En biologie cellulaire et du développement, il y a deux manières d'appliquer la chirurgie au laser. La première tire avantage des effets secondaires induits par l'expansion d'un 
plasma sur une échelle de quelques micromètres afin de détruire une cellule ou un groupe de cellules dans un organisme vivant en développement. Cette ablation est déjà possible en utilisant des impulsions longues de l'ordre de quelques nanosecondes et on parle alors encore de microchirurgie. La nanochirurgie, en revanche, s'adresse plus particulièrement à l'étude d'éléments subcellulaires. La destruction ciblée de structures subcellulaires ou d'organelles est ainsi possible sans affecter les parties superficielles (membranes) seulement si le procédé ablatif est parfaitement contrôlé, c'est-à-dire sans perturbation mécanique ou thermale. Être capable de modifier des structu- res cellulaires de l'ordre du nanomètre sans affecter la viabilité cellulaire a de nombreuses applications en biologie cellulaire et du développement ainsi qu'en procréation médicalement assistée, en particulier pour des études qui visent à comprendre le contrôle spatio-temporel des différentes étapes du développement, les interactions fonctionnelles entre différents organites, la dynamique du cytosquelette ou les échanges cellule-cellule.
A Morphogenèse : $D$. melanogaster



C Mécanique de positionnement du fuseau mitotique : S. Pombe

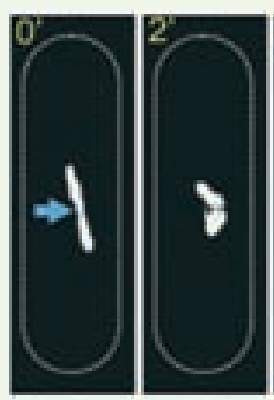

Écroulement


Perte

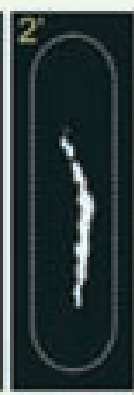

Courbure

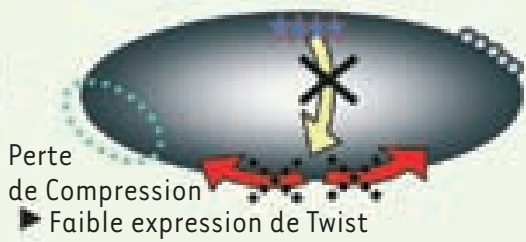

Faible expression de Twist



B Mécanique de positionnement du fuseau mitotique : $C$. elegans

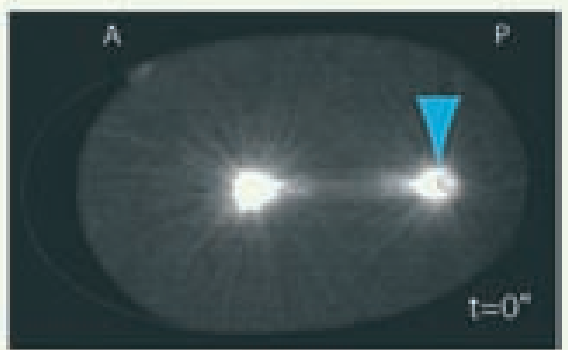

Avant irradiation

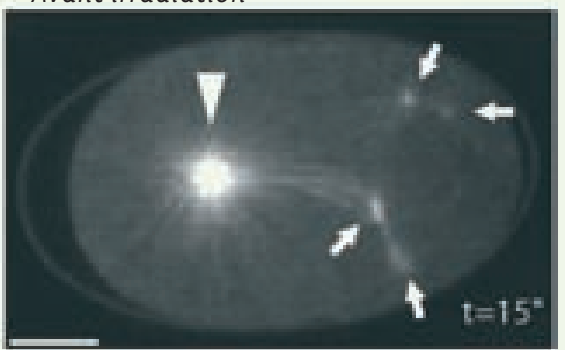

Après irradiation : traction des microtubules libérée
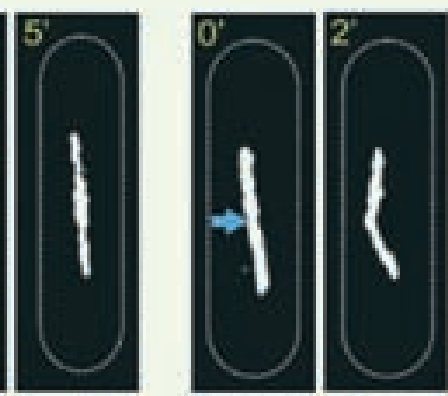

Croisement
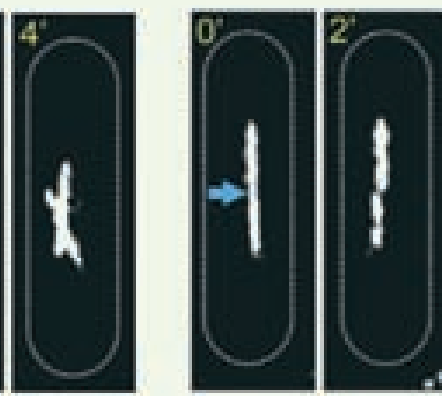

Glissement

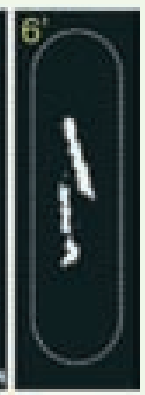

$$
\text { . }
$$

Figure 2. Études de forces à toutes les échelles. Les forces qui gouvernent l'organisation et l'évolution des tissus et des cellules peuvent être mises en évidence par la chirurgie laser. A. Supatto et al démontrent la perturbation des forces de morphogenèse chez la drosophile. Au cours de la gastrulation, l'embryon présente d'importants mouvements cellulaires couplés spatialement et temporellement (flèches jaunes et rouges). En utilisant un laser à femtosecondes, ces auteurs induisent la destruction de plusieurs cellules dorsales (à droite, flèches bleues) et bloquent les mouvements cellulaires latéraux (flèche jaune). La partie $\boldsymbol{A}$ montre une lignée transgénique exprimant la GFP et présentant un marquage fluorescent du contour cellulaire (images en microscopie de fluorescence à 2 photons, contraste inversé, reproduites avec la permission de Supatto et al. [16]). B. À une échelle subcellulaire, l'équilibre des forces établies autour du fuseau mitotique chez C. elegans peut être perturbé par un laser UV (470 ps par impulsion). En détruisant un pôle du fuseau (flèche bleue), les microtubules astraux interagissant avec le cortex tirent les résidus de pôle, montrant ainsi l'existence de forces de traction induites par les microtubules et responsables du positionnement du centrosome au centre de la cellule (d'après [8]). C. Chez la levure S. pombe, Tolic et al. démontrent les forces du fuseau en induisant des mécanismes de repositionnement du fuseau après dissection (laser à femtosecondes, flèches bleues). Images en fluorescence à 2 photons reproduites avec la permission de Tolic et al. [11]. Échelles: (A) $50 \mu \mathrm{m}$, (B) $10 \mu \mathrm{m}$. 
Biologie du développement et procréation médicalement assistée La biologie du développement analyse les étapes de différenciation (morphogenèse et organogenèse) qui donnent naissance à un individu à partir de la celluleœuf, aussi bien pour les plantes que les animaux. De nombreux modèles sont utilisés pour analyser les différentes étapes menant au développement complet d'un individu (D. melanogaster, C. elegans, A. thaliana). Des progrès considérables ont été accomplis dans la compréhension des phénomènes de développement chez ces différents organismes en utilisant des approches d'ablation cellulaire [14-18]. La plupart du temps, les méthodes de prédilection sont les aiguilles de micromanipulation dont la précision est de l'ordre du dixième de millimètre ou bien l'utilisation d'ARN messagers létaux sous le contrôle d'un promoteur tissu spécifique [19]. L'avènement de la microchirurgie laser a permis de détruire spécifiquement une cellule même si elle se situe à l'intérieur d'un embryon ou dans une région difficile d'accès car la manipulation ne nécessite en aucune manière de pénétrer jusqu'à la région d'intérêt. De plus, elle est extrêmement sélective car sa précision permet de ne détruire qu'une seule cellule ou un groupe de cellules. Le processus d'ablation de cellules durant le développement est illustré chez le nématode marin Platynereis dumerilii (Figure 1). En induisant la formation d'une série de plasmas au-delà du seuil énergétique de formation, on peut induire la vaporisation du milieu par cavitation, perméabilisant ainsi de manière irréversible les membranes cellulaires, et mener à l'ablation complète de plusieurs cellules précurseurs du développement d'organes spécifiques (Figure 1). L'intégrité de la membrane vitelline est généralement très bien préservée et des analyses, après développement ultérieur des embryons opérés, peuvent fournir de précieuses informations sur l'expression spécifique de gènes impliqués dans le développement de ces organes (par immunofluorescence ou hybridation in situ).

L'utilisation de la microchirurgie laser est aussi une des avancées les plus prometteuses dans le cadre de la fertilisation in vitro. Par un procédé identique de perméabilisation des membranes, de nombreuses manipulations sont possibles sur les ovocytes ou l'embryon pour augmenter le taux de fécondation et le taux d'implantation [20]. Le souci majeur est que toute manipulation peut engendrer des conséquences importantes sur le devenir de l'embryon, donc moins la technique est invasive, plus les risques à long terme sont faibles. Ainsi, l'utilisation de la microchirurgie au laser permet d'inciser de façon très précise les différentes couches de l'ovocyte ou de l'embryon, augmentant les chances de fertilisation et d'implantation et minimisant les effets secondaires [21].

\section{Biologie cellulaire}

Nous considérons trois modes d'utilisation de la nanochirurgie en biologie cellulaire, en fonction de la cible à étudier: ablation, dissection ou induction. La première est la simple miniaturisation de la chirurgie classique qui consiste à éliminer une partie de la cellule tout en préservant l'imperméabilité de sa membrane et d'observer, comme en biologie du développement, les mécanismes par lesquels la cellule réagit localement ou glo-

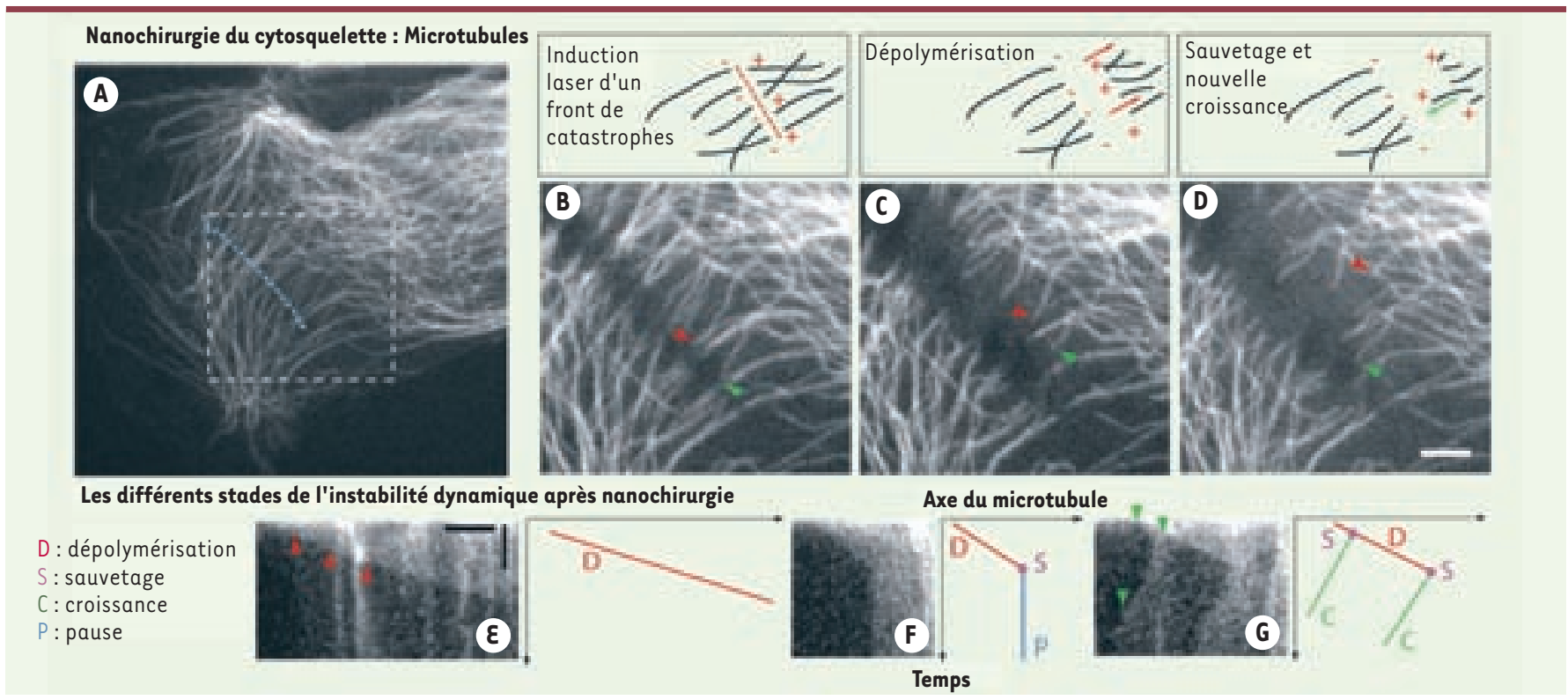

Figure 3. Nanochirurgie du cytosquelette en interphase. Dans les cellules de mammifères (A. cellule Ptk-2 transfectée de façon stable avec une construction $\alpha$ tubuline-YFP), les microtubules peuvent êtres sectionnés par un laser UV à impulsions (470 ps). L'irradiation à basse énergie (minimum $50 \mathrm{~nJ}$ ) le long d'une ligne (pointillés bleus) forme un front de catastrophes artificielles, créant ainsi de nouvelles pointes plus $(+)$ et moins $(-)$ des microtubules présents dans le volume de dissection. Chaque microtubule réagit de manière différente. La dépolymérisation (flèches rouges en $B, C, D, \varepsilon$ ) de la pointe $(+)$ est le phénomène le plus fréquent après la catastrophe et le sauvetage alors que la pointe (-) reste relativement stable. La dépolymérisation, la recroissance ou l'état de pause après une catastrophe au laser sont illustrés en $\varepsilon, F$ et $G$ à l'aide de kymographes (graphe traçant l'intensité lumineuse le long de l'axe du microtubule en fonction du temps). Les flèches vertes en $G$ suivent la progression de la pointe d'un microtubule au travers des phases de dépolymérisation, sauvetage et recroissance en correspondance avec la séquence d'images $B, C, D$. L'instabilité dynamique de simples filaments peut être induite et mesurée n'importe où dans le cytoplasme (adapté d'après [4]). Échelle : (D) $5 \mu \mathrm{m}$, (E) $5 \mu \mathrm{m}$ horizontal, 30 secondes vertical. 
balement. Ainsi, il est possible de détruire spécifiquement des mitochondries [6], un centriole [22] ou une neurite [23, 24]. La dissection permet d'avoir accès à des structures cellulaires normalement masquées. On peut ainsi accéder à la membrane plasmique des cellules de plantes pour effectuer des mesures par patch-clamp [25] ou y insérer des bactéries [26]. La dissection à l'échelle intracellulaire permet également de disséquer des sous régions chromosomiques, comme démontré par König et al. [13] qui ont atteint une précision chirurgicale de $110 \mathrm{~nm}$ sur des chromosomes humains. L'induction concerne plus spécifiquement l'étude de phénomènes dynamiques. L'utilisation du laser permet d'induire des modifications structurales et de changer l'équilibre dynamique de façon ciblée et non invasive, permettant l'observation du comportement hors équilibre ou du retour vers l'équilibre. Cela concerne les différents éléments du cytosquelette [4], les flux cytoplasmiques [27], la motilité cellulaire et la morphogenèse [16-18].

\section{Étude dynamique de forces}

Des études récentes ont démontré des applications très élégantes de la chirurgie au laser in vivo. Chez la drosophile ( $D$. melanogaster), au cours de la gastrulation, l'embryon présente d'importants mouvements cellulaires couplés spatialement et temporellement (Figure 2A). Supatto et al. [16] ont pu ainsi démontrer que la destruction de plusieurs cellules dorsales à l'aide d'un laser à femtosecondes permet de bloquer les mouvements latéraux et de perturber l'intégrité mécanique de l'ensemble de l'embryon. En particulier, les cellules du pôle antérieur présentent un comportement mécanique très différent après une ablation laser. Ce comportement mécanique a été corrélé avec une variation de l'expression mécano-sensible de la protéine Twist. D'autres auteurs ont appliqué la chirurgie laser au mécanisme de fermeture dorsale de la drosophile [17, 18].

Au niveau subcellulaire, afin de révéler la nature des forces exercées sur le fuseau mitotique pour permettre son positionnement, Grill et al. [28] ont sectionné la partie médiane ou un pôle du fuseau mitotique lors de la première division asymétrique chez le nématode C. elegans (Figure $2 B$ ). Perturbant ainsi l'équilibre des forces établies autour des pôles, ils démontrent l'action de traction des microtubules astraux vers le cortex et la distribution asymétrique de cette traction, suggérant que l'asymétrie nécessaire pour engendrer deux cellules sœurs de tailles différentes est induite par le cortex par le biais des microtubules. Cette destruction de l'intégrité du fuseau mitotique a été obtenue à l'aide d'un laser ultraviolet dont la durée d'impulsion dépassait la nanoseconde. Une expérience similaire a été menée peu après, à une échelle encore plus réduite, dans la levure S. pombe par Tolic et al. [11] (Figure 2C) et Khodjakov et al. [29]. Ces auteurs utilisent un laser identique pour observer le signal de fluorescence du fuseau mitotique en anaphase et le perturber en utilisant une énergie lumineuse sensiblement plus élevée pour obtenir l'effet chirurgical. Induisant une fracture du fuseau d'une largeur estimée à 200 nm en sa partie médiane, ils distinguent différentes réactions telles que le repliage, l'écroulement, le croisement ou le glissement du fuseau, phénomènes témoignant des forces de positionnement au centre de la cellule.

\section{Vers une dynamique « régionale » du cytosquelette}

Les constituants du cytosquelette sont des polymères dynamiques très réactifs aux effets du laser à des intensités bien inférieures au seuil de formation du plasma (Encart 1). Cette propriété permet d'induire des modifications de ces polymères avec une précision allant jusqu'au simple filament ou microtubule et d'en étudier la dynamique, les tensions ou les forces en présence.

Que ce soit chez la levure S. pombe, le nématode C. elegans ou les cellules de mammifères, il est possible de couper spécifiquement certains microtubules. Les microtubules sont des polymères cylindriques creux constitués de dimères de tubuline qui oscillent constamment entre des phases de polymérisation, catastrophe, dépolymérisation et sauvetage. Cette propriété de polymérisation hors équilibre a été baptisée instabilité dynamique [30]. La césure des microtubules par le laser [31] représente une catastrophe artificiellement induite qui ne modifie pas les principes de l'instabilité dynamique, comme nous l'avons récemment démontré (Figure 3). L'avantage de la nanochirurgie laser est de permettre d'induire cette catastrophe à n'importe quel endroit de la cellule, en contraste avec les études classiques uniquement possibles via l'utilisation de drogues (nocodazole) qui perturbent de manière globale l'organisation cellulaire, ou avec les études en microscopie à fluorescence possibles seulement dans des régions cellulaires limitées. Par conséquent, la mesure directe des paramètres de l'instabilité dynamique des microtubules liés à des organites (mitochondrie, chloroplaste, appareil de Golgi) ou à des structures cellulaires (points d'adhérence, réticulum endoplasmique transitoire) est maintenant possible et peut conduire à une approche locale ou régionale de l'étude du cytosquelette.

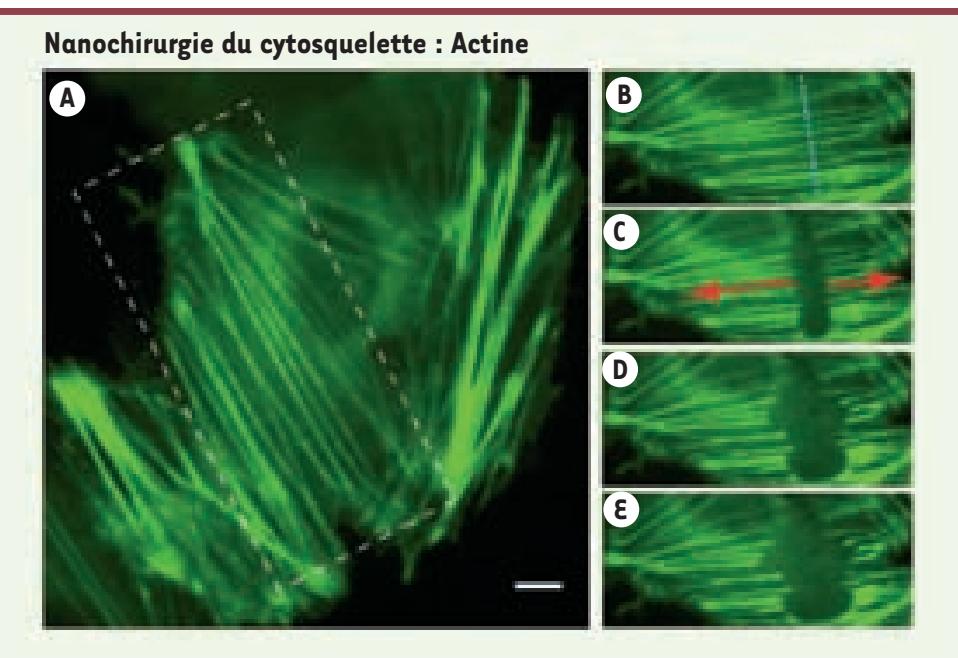

Figure 4. Relâchement de tension des fibres de stress. Les fibres de stress établissent des forces de contraction entre les points d'adhésion des cellules en culture. Cellule de mammifère Ptk-2 transfectée avec actine-GFP. En appliquant la nanochirurgie avec un laser pulsé (470 ps, 100 nJ par impulsion) le long de la ligne pointillée bleue $(B$, vue extraite de l'aire en pointillés de $A$ ), on observe une rétraction de ces fibres en quelques secondes ( 30 secondes de $\boldsymbol{B}$ à $\boldsymbol{\varepsilon}$ ), suggérant le relâchement des forces de contraction (adapté d'après [4]). Échelle (A) : $5 \mu \mathrm{m}$. 
De même, les fibres de tension d'actine, ou fibres de stress, peuvent être sectionnées (Figure 4). Témoignant de la tension qu'une cellule exerce sur l'environnement externe, les fibres de stress s'étendent en général entre les points d'adhésion. En découpant ces fibres, nous avons montré que ces dernières se rétractent, suggérant ainsi le relâchement des forces d'adhésion exercées par la cellule. Les fibres de stress sont des structures très hétérogènes et stables au cours du temps. La nanochirurgie permet donc de mettre en évidence la dynamique, en apparence invisible car à l'équilibre, des forces en jeu au cours du processus d'adhérence cellulaire.

\section{Conclusions}

Ainsi, la nanochirurgie laser ouvre de larges possibilités d'étude en biologie cellulaire et du développement que ce soit par ablation ou destruction d'organites ou de sous-unités fonctionnelles de la cellule ou par la perturbation des forces régissant la dynamique du cytosquelette in vivo. De plus, les modifications qu'engendre le plasma sont d'une grande précision et ont une portée limitée autour d'une région cellulaire définie, permettant des études localisées et non invasives des forces en présence. $\diamond$

\section{REMERCIEMENTS}

Les auteurs remercient F. Nédélec pour ses précieux commentaires sur le texte, I. Tolic, W. Supatto, A. Vogel, S. Klaus et D. Arendt pour leurs contributions au contenu de ce manuscrit. Ce travail a été partiellement financé par le BMBF, ministère allemand de l'éducation et de la recherche.

\section{SUMMARY}

\section{Laser nanosurgery in cell biology}

Since their first use in the early 60's, pulsed lasers have become increasingly popular for their ability to ablate biological tissue. Short laser pulses allow high precision surgery for biological and medical applications with minimal invasiveness. Performing highly targeted manipulation and ablation allows experiments impossible so far in development biology, cellular biology or even assisted reproductive technologies and laser surgery has been increasingly used over the last five years to answer key questions in Biology. Recently, picosecond UV and femtosecond IR laser pulses have been used to cleave microtubules and to severe actin stress fibers in vivo with a spatial precision in the submicrometer range to study their dynamics without affecting cell viability. We review recent findings on the underlying principles of pulsed laser nanosurgery mechanisms showing how the use of ultra short laser pulses increases precision and non-invasiveness of laser surgery. We show how the understanding of the surgical process allows one to distinguish between single cell ablation in living organisms or intracellular nanosurgery in living cells and we review recent applications to the study of forces and the quantification of cytoskeleton dynamics. $\diamond$

\section{RéFÉRENCES}

1. Vogel A, Venugopalan V. Mechanisms of pulsed laser ablation of biological tissues. Chem Rev 2003; $103: 577-644$

2. Niemz M. Laser-tissue interactions. Fundamentals and applications, $2^{\text {nd }}$ ed. Berlin : Springer Biological and Medical Physics Series, 2002.

3. Brunetaud JM, Mordon S, Desmettre T, Beacco C. In : Fabre C, Pocholle JP, eds. Les lasers et leurs applications scientifiques et médicales. Les Ulis : EDP Sciences, 1996-2002.

4. Colombelli J, Reynaud $\varepsilon G$, Rietdorf J, et al. In vivo selective cytoskeleton dynamics quantification in interphase cells by pulsed UV laser nanosurgery. Traffic $2005 ; 6$ : 1093-102.
5. Botvinick EL, Venugopalan V, Shah JV, et al. Controlled ablation of microtubules using a picosecond laser. Biophys J 2004 ; 87 : 4203-12.

6. Watanabe W, Arakawa N, Matsunaga S, et al. Femtosecond laser disruption of subcellular organelles in a living cell. Opt Exp $2004 ; 12$ : 4203-13.

7. Srinivasan R. Ablation of polymers and biological tissue by ultraviolet lasers. Science $1986 ; 234: 559$.

8. Colombelli J, Grill SW, Stelzer EHK. Ultraviolet diffraction limited nanosurgery of live biological tissues. Rev Sci Instr 2004 ; 75 : 472-8.

9. Joglekar AP, Liu HH, Meyhöfer $\varepsilon$, et al. Optics at critical intensity : applications to nanomorphing. Proc Natl Acad Sci USA 2004 ; 101 : 5856-61.

10. Vogel A, Noack J, Hüttman G, Paltauf G. Mechanisms of femtosecond laser nanosurgery of cells and tissues. Appl Phys B 2005; 81 : 1015-47.

11. Tolic-Nørrelykke IM, Sacconi L, Thon G, Pavone FS. Positioning and elongation of the fission yeast spindle by microtubule-based pushing. Curr Biol 2004 ; $14: 1181-6$.

12. Heisterkamp A, Maxwell IZ, Mazur $\varepsilon$, et al. Pulse energy dependence of subcellular dissection by femtosecond laser pulses. Opt Exp 2005 ; $13: 1390-6$.

13. Koenig K, Riemann I, Fritzsche W. Nanodissection of human chromosomes with near-infrared femtosecond laser pulses. Opt Lett $2001 ; 26: 819-21$.

14. Montell DJ, Keshishian H, Spradling AC. Laser ablation studies of the role of the Drosophila oocyte nucleus in pattern formation. Science 1991 ; $254: 290-3$.

15. Berger F. Cell ablation studies in plant development. Cell Mol Biol (Noisy-leGrand) 1998; $44: 711-9$.

16. Supatto W, Debarre D, Moulia B, et al. In vivo modulation of morphogenetic movements in Drosophila embryos with femtosecond laser pulses. Proc Natl Acad Sci USA 2005 ; 102 : 1047-52.

17. Hutson MS, Tokutake Y, Chang MS, et al. Forces for morphogenesis investigated with laser microsurgery and quantitative modelling. Science 2003; $300: 145-9$

18. Kiehard DP, Galbraith CG, Edwards KA, et al. Multiple forces contribute to cell sheet morphogenesis for dorsal closure in Drosophila.J Cell Biol 2000 ; 149 : 471-90.

19. Rulifson $\varepsilon$ J, Kim SK, Nusse R. Ablation of insulin-producing neurons in flies : growth and diabetic phenotypes. Science 2002 ; 296: 1118-20.

20. Schöpper B, Ludwig M, Edenfeld J, et al. Possible applications of lasers in assisted reproductive technologies. Hum Reprod. 1999 ; 14 (suppl 1) : 186-93.

21. Blanchet GB, Russell JB, Fincher CR Jr, Portmann M. Laser micromanipulation in the mouse embryo : a novel approach to zona drilling. Fertil Steril 1992 ; $57: 1337-41$.

22. Khodjakov A, Cole RW, Oakley BR, Rieder CL. Centrosome-independent mitotic spindle formation in vertebrates. Curr Biol $2000 ; 10: 59-67$.

23. Gross GW, Lucas JH, Higgins ML. Laser microbeam surgery : ultrastructural changes associated with neurite transection in culture. J Neurosci 1983 ; 3: 1979-93.

24. Yanik MF, Cinar $\mathrm{H}$, Cinar $\mathrm{HN}$, et al. Neurosurgery : functional regeneration after laser axotomy. Nature $2004 ; 432: 822$.

25. Henriksen GH, Taylor AR, Brownlee C, Assmann SM. Laser microsurgery of higher plant cell walls permits patch-clamp access. Plant Physiol 1996; 110 : 1063-8.

26. Buer CS, Gahagan KT, Swartzlander GA Jr, Weathers PJ. Insertion of microscopic objects through plant cell walls using laser microsurgery Biotechnol Bioeng $1998 ; 60: 348-55$.

27. Hahne G, Hoffmann F. The effect of laser microsurgery on cytoplasmic strands and cytoplasmic streaming in isolated plant protoplasts. Eur J Cell Biol 1984 ; $33: 175-9$.

28. Grill SW, Gonczy P, Stelzer EHK, Hyman AA. Polarity controls forces governing asymmetric spindle positioning in the Caenorhabditis elegans embryo. Nature $2001 ; 409: 630-3$.

29. Khodjakov A, La Terra S, Chang F. Laser microsurgery in fission yeast : role of the mitotic spindle midzone in anaphase B. Curr Biol $2004 ; 14: 1330-40$.

30. Mitchison TJ, Kirschner M. Dynamic instability of microtubule growth. Nature $1984 ; 312: 237-42$

31. Tao W, Walter RJ, Berns MW. Laser-transected microtubules exhibit individuality of regrowth, however most free new ends of the microtubules are stable. J Cell Biol 1988; 107 : 1025-35.

\section{TIRÉS À PART}

J. Colombelli 